\title{
Téoros
}

Revue de recherche en tourisme

\section{Tourisme sur neige : du ski de fond au tourisme d'aventure}

\section{Henri Jamet}

Volume 8, numéro 3, novembre 1989

Tourisme hivernal

URI : https://id.erudit.org/iderudit/1080310ar

DOI : https://doi.org/10.7202/1080310ar

Aller au sommaire du numéro

Éditeur(s)

Université du Québec à Montréal

ISSN

0712-8657 (imprimé)

1923-2705 (numérique)

Découvrir la revue

Citer cet article

Jamet, H. (1989). Tourisme sur neige : du ski de fond au tourisme d'aventure.

Téoros, 8(3), 26-28. https://doi.org/10.7202/1080310ar d'utilisation que vous pouvez consulter en ligne.

https://apropos.erudit.org/fr/usagers/politique-dutilisation/ 


\section{Tourisme sur neige: Du ski de fond au tourisme d'aventure}

Avec une couverture nivale de 1640000 $\mathrm{km}^{2}$ qui persiste bon an, mal an, pendant cinq mois par année à la hauteur du $45^{\circ}$ parallèle, huit mois par année à la hauteur du $62^{\mathrm{e}}$ parallèle, nous devons admettre que le Québec a été choyé par les dieux et qu'il est bien pourvu pour se tailler un créneau généreux dans le lucratif marché du tourisme hivernal.

\section{La garantie neige}

Elle est aussi essentielle au succès de l'opération, que le positionnement des espaces blanes.

À ce niveau, on doit rendre grâce en premier à Voltaire qui attira l'attention mondiale sur la vocation hivernale du Québec en faisant passer à l'histoire "les quelques arpents de neige de la Nouvelle-France". Plus près de nous, Émile Nelligan nous entraîne dans la magie de ses jardins de givre avec son poème "Soir d'hiver". Ce fut pourtant Gilles Vigneault, l'un des grands chantres de la révolution tranquille, qui n'hésita pas à faire de l'hiver québécois un pays pour tous les gens de la terre avec son hymne "Mon pays, c'est l'hiver!" Le journaliste Georges-Hébert Germain, pour contrecarrer les effets ravageurs d'uncertain

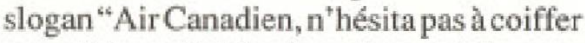
pour l'Actualité le titre d'un article pour promouvoir le trekking dans le Grand Nord québécois d'un nouveau slogan non équivoque "Mon anorak, ma brosse à dents! on y va, on y va!" La Province de Québec se souvient de ceux qui ont fait connaître ses hivers.
Si l'hiver a connu quelques ratées au cours de certaines saisons au grand désespoir des skieurs alpins, les stations de ski n'ont pas tardé à répondre à cet affront en décidant de "prendre leur neige en main" en se dotant de systèmes sophistiqués de neige artificielle. Si le nom de Armand Bombardier est désormais associé pour toujours à la motoneige, il en sera probablement ainsi sur Louis Enfield, ingénieur du Québec, qui a tiré profit des défaillances du système pour fabriquer le "TurboCristal", le canon à neige le plus performant au monde.

Lorsque la neige fut vaincue, les Tours Operators et les stations de ski québécoises soulagés ajoutèrent à leur promotion ce label de "garantie neige" que recherchent encore désespérément certaines grandes stations de sports d'hiverà travers lemonde.

\section{Les produits vedettes de l'hiver}

Il y en a trois qui ont atteint une certaine maturité: ski de fond, ski alpin, motoneige et un quatrième qui cherche à se tailler une place au soleil, mais les pieds dans la neige, le tourisme d'aventure.

\section{Le ski alpin (faits saillants)}

Il fait partie des huit produits priorisés par le ministère du Tourisme dans son nouveau plan de stratégie et de développement (Samson Bélair, planification 1989-1992). Il a bénéficié d'une aide importante grâce en partie à l'entente auxiliaire CanadaQuébec sur le ski alpin qui était destinée à rééquiper les centres de ski majeur de plus de 300 mètres de dénivellation. Les 846 000 skieurs ont fait 8545000 jours/ski au cours de lasaison 1985/1986. Ils ont dépensé 296 millions de dollars en dépenses directes. Les $2 / 3$ de ces dépenses ont été effectuées par ceux qui utilisent l'hébergement commercial.

\section{Le ski de randonnée (faits saillants)}

Il ne fait pas partie des huit produits priorisés par le ministère du Tourisme, mais il peut trouver une certaine place dans le cadre du tourisme d'aventure.

Les 417000 skieurs de fond ont effectué au cours de l'hiver 1985-1986, 14700000 jours/ski. Six cent un mille skieurs de fond, soit $42,4 \%$ sont des skieurs occasionnels tandis que 332000 , soit $23,4 \%$ sont des skieurs réguliers.

Les dépenses directes découlant de la pratique du ski de fond avec 272 millions de dollars sont presque équivalentes à celles du ski alpin (296 millions) pour la saison 1985-1986.

\section{Motoneige (faits saillants)}

Ceproduit fait partiedes huit produits priorisés par le ministère du Tourisme. Les 513 000 motoneigistes ont effectué 5387000 jours/motoneige au cours de l'hiver 19851986. Les 259000 pratiquants réguliers représentent $50,5 \%$ des utilisateurs, 239 000 personnes sont propriétaires de leur véhicule. Un motoneigiste sur cinq utilise l'hébergement commercial, c'est la région des Laurentides qui est la plus populaire suivi par l'Outaouais.

Globalement, les dépenses directes découlant de la pratique de la motoneige seraient de l'ordre de 141 millions, comparativement à 272 millions pour le ski de randonnée et de 296 millions pour le ski alpin (hiver 1985-1986),

La motoneige a surtout été jusqu'à présent considérée comme une activité récréative, plutôt que touristique, malgré le fait qu'une dizaine de compagnies de location de motoneige mettront à la disposition de la clientèle locale et internationale 400 véhicules l'hiver prochain. C'est le rallye "Harricana ler Fuji extrême, un fantastique raid en motoneige au pays des aurores boréales" qui va consacrer ce produit touristique au niveau international. La nouvelle officielle a été annoncée à la suite du ler raid de reconnaissance de 13 motoneiges qui s'est tenu du 18 février au 13 mars 1989 sur une distance de $3660 \mathrm{~km}$ entre Montréal et

"Monsieur Henri Jamet, ministere du Tourisme 
Radisson en passant par Québec, Pointeau-Pic, Roberval, Chibougamau, Némis-

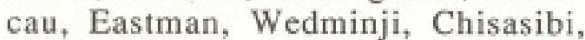
Kuujjuarapik. L'idée de ce projet original revient à Nicolas Hulot, l'animateur de l'émission hebdomadaire de l'aventure de TF1 "Ushuaîa" qui rejoint chaque semaine plus de six millions de fans. Nicolas Hulot s'est entouré de grands noms comme René Metge, quatre fois vainqueurduParis Dakar, Thierry Reverchon, un des pères du Paris Dakar, Hubert De Chevigny, qui a fait le pôle nord en ULM, Jean-Claude Bibollet qui a fait une expédition dans les Torngat en 1981, au Yukonen 1984, Pierre Le Nomand qui compte plusieurs expéditions dans l'Artique.

\section{TF1 crée l'événement}

Comme dit Xavier Couture: "Chez TF1, nous mettons le paquet", c'est-à-dire plus de deux heures et demie $\mathrm{d}^{*}$ antenne pour Harricana, des images télédiffusées dans trente-sept pays, seize pages sur FigaroMagazine, etc. Cet exercice de repérage a eu des retombées médiatiques de cinq millions.

TF1 est absolument décidé de faire de ce raid des neiges qui débutera officiellement l'année prochaine une opération de très grande envergure comparable au Paris Dakar. Pour 1990 sont attendus 240 participants, 180 joumalistes du monde entier, une Equipe de soutien de 100 personnes, quelques centaines ou milliers de touristes de l'extérieur et des retombées médiatiques beaucoup plus élevées que celles du printemps dernier.

\section{Tourisme d'aventure d'hiver}

Ce produit touristique est bien implante au Groënland, au Spitsberg en Iceland, dans le Nord des pays scandinaves, dans les Territoires du Nord-Ouest au Yukon, en Alaska, au Labrador. Même la presqu'île soviétique de Kola, situce à l'Est de la Laponie finlandaise, vient d'ouvrir sa frontière qui était fermée hermétiquement par l'Armée rouge. Le tourisme d'aventure ne connaît plus de frontière.

Ce produit qui était perçu comme une activité spectaculaire, mais marginale au Québec, vient d'être reconnu par le ministère du Tourisme parmi les huit produits priorisés. Il est considéré comme produit d'appel, produit d"avant-garde à développer, à mettre en marché.

La traversée du désert est terminée pour le tourisme d'aventure qui permet de générer des retombées économiques importantes dans les régions éloignées des grandes villes. L'Abitibi-Témiscamingue, les Territoires de la Baie-James, le Grand Nord québecois, la région de Duplessis se préparent à relever le defi.

On a utilisé dans ce texte à quelques reprises le terme "trekking" qui est le synonyme de tourisme d'aventure. Le "Petit Robert" donne comme définition à trekking: randonnée touristique dans des régions touristiques difficilementaccessibles. C'estGilles Parent, guide de montagne québécois qui inaugura les expeditions faites par des professionnels de l'aventure lors de la traversée Est-Quest de la chaîne des Torngat en 1973, en ski de randonnée.

C'est l'Italien Beppi Tenti, guide de montagne, président-directeur général de la Cie de trekking de Turin, Alpinismus Intemational, qui commercialisa en 1973 les premiers forfaits de traîneaux à chiens entre Kangirsuk et Kuujuag dans le Grand Nord québécois, pour la riche clientèle du Jet set international du Nord de l'Italie.

"Les touristes italiens désiraient s'intégrer à la vie des Inuits, s'habiller comme eux, manger comme eux, domir dans des igloos et voyager comme autrefois en kamutik, tirés par une meute de chiens."

Le Cépal de Jonquière vendit les premiers forfaits de ski de randonnée et de raquette dans les Monts Othis, de ski de fond, traîneaux à chiens dans les Monts Groulx et la Corporation de développement des Naskapis, les premiers raids en motoneige de Schefferville à Kuujuaq.

\section{Les produits du tourisme d'aventure hivernal québécois}

- raid en traîneau à chiens; raid en motoneige;

- raid en ski de randonnée;

- raid en quatre roues motrices (où l'activite est autorisé);

- raid en ski de fond associé au traîneạ à chiens:

- raid en raquettes;

- randonnées pédestres pour observer autour des villages les lagopèdes des saules ou des roches dans leur livré d'hiver, les bruants des neiges, les caribous, le vol des outardes, des oies blanches, 1 "harfang des neiges, les ébats des bellugas dans la baie de Ivujivik.

\section{Destinations du trekking}

Le tourisme d'aventure se nourrit de grands espaces, dont les limites sont situées en marge de l'écoumène. Les destinations forfaits haut de gamme sont les Territoires de la Baie-James, le Grand Nord québécois et le Labrador.

Les forfaits de moyenne gamme sont l'Abitibi, le Témiscamingue, la zone de Chibougamau, la Basse-Côte-Nord, Anticosti et les territoires peu peuplés. Les sites recherchés sont les Monts Groulx, Les Monts Othis, les Torngat, le cratère du Nouveau-Québec, le Golfe Richmond.

Ces destinations permettent de découvrir, de partager la vie de peuples de cultures différentes: Inuit, Montagnais, Algonquins, Naskapis, Cris. Lecontact avec ces peuples est une des composantes importante essentielle du tourisme d'aventure, qui est un tourisme de découverte humaine, autant que de découverte de la nature (faune, flore, habitats fauniques).

\section{Le marché}

Les marchés présentant le plus haut potentiel sont: la France, les U.S.A., l'Allemagne, la Suisse, le Danemark, 1'Angleterre, I'Italie (Nord), le Canada (Ontario et Québec).

\section{Le profil de la clientèle}

D'après Doyle N.Y. Travel Data Market Facts, il y a environ le tiers de la clientèle touristique américaine qui correspond au profil type de la clientèle du tourisme d'aventure.

$D^{\dagger}$ après une enquête du grossiste français Delta, la clientèle du trekking comprend 5 $\%$ d'étudiants, $19,5 \%$ de professions liberales, $24 \%$ d'enseignants, $41 \%$ de fonctionnaires, $4 \%$ de retraités. C"est la clientèle de 21 aे 49 ans qui constitue $80 \%$ de la clientèle. Elle veut vivre 1'Aventure avec un grand $\mathrm{A}_{\text {, }}$ elle veut vivre l'Evasion avec un grand É, synonyme de liberté. D'après Gault et Millau, (ref. no 57) ${ }^{4}$ Les vacances aventures: c"est découvrir en profondeur d'autres terres, d'autres humains; c'est de touriste spectateur, voyeur, passer au niveau de TOURISTE/ACTEUR ${ }^{*+}$.

D'après une enquête du MICT, le tourisme d'aventure recrute une bonne partie des "fans" chez les enseignants, les infirmières, les salariés moyens. Enfin la clientèle âgée de 18 à 35 ans représente de 50 à $60 \%$ de 1 'ensemble. 


\section{Mise en marché}

Pour organiser ces voyages insolites, une nouvelle "race de Tours Operators" très spécialisée $s^{7}$ est constituée à travers le monde. Certains offrent les destinations neiges et glaces. Les principaux T.O. neiges et glaces sont: Agrepa Erik le Rouge, Atlant's Tours, Alibert, Andestour, Atalante, Bennett Voyage, Canadian National, Club Aventure, Deserto, Explorator, Culliver, Itinérances, La Burle, Le Renard Vagabond, Peuples du Monde, Terres d'Aventure, Visages, Visages Natures. Nouveau-Monde, Aventures Arctiques, Nouvelles Frontières, Terres lointaines, Le Renard Vagabond, Grand-Nord, GrandLarge, CIAM, Atalante et Alibert proposent des forfaits trekking hivernaux au Québec. Il y a plus de 300 Tours Operators toutes destinations à travers le monde.

\section{Fabricants de produits trekking}

Il y a une vingtaine de fabricants de forfaits de trekking au Québec. Ce sont pour l'instant de petites compagnies qui grossissent généralement année après année.

Elles ont de plus en plus tendance à faire commercialiser leurs forfaits par des grossistes bien établis sur les marchés québécois, français, suisse, belge et américain. Ces producteurs se sont réunis à Sept-Iles en mai dernier lors du premier colloque sur le tourisme d'aventure. Lors de cette rencontre, ils ont créé une association pour les regrouper et les représenter.

Le Ministère, de son côté, a nommé deux responsables du produit tourisme d'aventure. Le responsable du développement de l'offre, Henri Jamet et le chef de produit mise en marché, Michel Leblanc. Le ministère du Tourisme a également créé un comité mixte "Tourisme d'aventure" qui devrait se réunir incessamment. Il est composé de onze personnes représentant le ministère du Tourisme, les fabricants, les Associations touristiques et régionales, le ministère du Loisir, de la Chasse et de la Pêche.

\section{Perspectives d'avenir pour le tourisme hivernal}

Les activités de ski connaissent un développement important en particulier le ski alpin depuis que l'entente auxiliaire Canada-Québec a permis de moderniser cette industrie qui souffrait d'un certain ralentissement. Le produit motoneige a surtout été considéré comme une activité récréative jusqu’à présent. Harricana va lui donner une orientation plus touristique que récréative et entraîner dans ses traces un nouveau positionnement de l'hiver québécois où le tourisme d'aventure va pouvoir se tailler une place enviable. Le Yukon avec une superficie de $478000 \mathrm{~km}^{2}$ et une populationde 27000 habitants est unmodèle de référence: ces 56 pourvoyeurs de trekking vous offriront l'aventure de votre vie. Il y a maintenant autant de pourvoyeurs de trekking que dechasseet de pêche au Yukon. Certains offrent ces trois activités. Le Yukon offre une capacité d'hébergement de 2000 chambres.

Malgré un été très court, le Yukon a reçu l'année dernière 480000 visiteurs; l'Alaska 700 000; la Laponie finlandaise $875000_{4}$ l'Islande 129000 ; le Grand Nord québécois et la Baie-James 20 000; le Groenland 50 000 (le village de Jakobshaven avec 6000 chiens traîneaux ne fournit même plus à la demande touristique); le Labrador 10000 et les Territoires du Nord Ouest 50000 environ.

Les déserts blancs ne sont pas moins vierges, moins rudes ni moins riches d'émotion que les déserts de sable. De la Patagonie aux confins du Pôle Nord, du Grand Nord québécois aux Territoires du Nord-Ouest, à pied, en ski, en traîneau à chiens, en raquettes, en quatre roues motrices, en motoneige, en avion sur ski, en traîneau tiré par des rennes, ils s'offrent à toutes les explorations, à tous les rêves des biens nantis.

Notes explicatives

1- Cluzeau, Patrick, Sondage omnibus, le tourisme hivernal des québécois: les skieurs alpins, les motoneigistes, les skieurs de randonnee, saison d'hiver 1985-1986; MTO, décembre 1986.

2. Jamet, Herri, Le tourisme d'aventure, trokking. etude du produit, étude de marché, plan de marketing. MTO, mai 1985.

3- Le Hoverou, Alexandrine, Action Canada France, Harricana 1er Fuji extrëme, avril 1989 